Article

\title{
Isomorphic Classification of Reflexive Müntz Spaces
}

\author{
Sergey V. Ludkowski
}

Department of Applied Mathematics, Moscow State Technological University (MIREA), av. Vernadsky 78, Moscow 119454, Russia; Ludkowski@mirea.ru

Received: 6 November 2017; Accepted: 5 December 2017; Published: 18 December 2017

Abstract: The article is devoted to reflexive Müntz spaces $M_{\Lambda, p}$ of $L_{p}$ functions with $1<p<\infty$. The Stieltjes transform and a potential transform are studied for these spaces. Isomorphisms of the reflexive Müntz spaces fulfilling the gap and Müntz conditions are investigated.

Keywords: Banach space; Müntz space; reflexive; isomorphism; Schauder basis; Stieltjes transform

\section{Introduction}

A function approximation and an isomorphic classification of Banach spaces play a very important role in functional analysis and in geometry (see, for example, literature from [1-19] and references therein). Among Banach spaces, a large class consists of Müntz spaces (see [1,4,9,17,20,21] and references therein).

The reflexive Müntz spaces $M_{\Lambda, p}(\mathbf{F})$ are defined as completions of a F-linear span of the monomials $t^{\lambda}$ with $\lambda \in \Lambda$ on the segment $[0,1]$ relative to the $L_{p}$ norm, where $\Lambda \subset[0, \infty), t \in[0,1]$, $1<p<\infty$, where $\mathbf{F}$ is either the real field $\mathbf{F}=\mathbf{R}$ or the complex field $\mathbf{F}=\mathbf{C}$. It is worth mentioning that generally monomials $t^{\lambda}$ do not form a Schauder basis in the Müntz space $M_{\Lambda, p}$. For a long time, whether or not they have Schauder bases remained a problem $[1,2,8,16]$.

This article is devoted to the reflexive Müntz spaces $M_{\Lambda, p}$ fulfilling the gap and Müntz conditions. For this purpose, the Stieltjes transform and a potential transform are studied (see Propositions 1 and 2 and Corollary 2). This study is based on certain useful properties of the Fourier transform in the reflexive Müntz spaces with a change of the variable (Lemmas 4, 5, and 6 and Corollary 1). Their Banach space geometry is investigated in Propositions 3 and 4 and Theorem 1. A relation with the Banach space $l_{p}=l_{p}(\mathbf{F})$ over the field $\mathbf{F}$ is elucidated.

It is proven in Theorem 1 that under the aforementioned conditions $M_{\Lambda, p}(\mathbf{F})$ is isomorphic with $l_{p}(\mathbf{F})$.

All main results of this paper are obtained for the first time. They can be used for further studies of Banach space geometry, measures and stochastic processes in Banach spaces, approximations of functions.

\section{The Müntz $M_{\Lambda, p}$ Spaces}

To avoid misunderstandings, we first give our notation and some useful Lemmas 1-3.

Notation 1. As is usual, $L_{p}(\Omega, \mathcal{F}, v, \mathbf{F})$ denotes the Banach space of all $v$-measurable functions $f: \Omega \rightarrow$ F having a finite norm

$$
\|f\|_{L_{p}(\Omega, \mathcal{F}, v, \mathbf{F})}:=\left(\int_{\Omega}|f(x)|^{p} v(d x)\right)^{1 / p}<\infty,
$$

where $1<p<\infty$ is a marked number, $\mathcal{F}$ is a $\sigma$-algebra of subsets in a set $\Omega, v$ is a $\sigma$-finite nonnegative measure on $\mathcal{F}$, either $\mathbf{F}$ is the real field $\mathbf{R}$ or $\mathbf{F}$ stands for the complex field $\mathbf{C}$. Then, the closure of the F-linear span $T:=c l-\operatorname{span}_{\mathbf{F}}\left\{t^{k}: t \in[0,1] ; k \in \Lambda\right\}$ in the Banach space $L_{p}([0,1])=$ $L_{p}([0,1], \mathcal{B}([0,1]), \mu, \mathbf{F})$ is called the Müntz space $M_{\Lambda, p}:=M_{\Lambda, p}([0,1], \mathcal{B}([0,1]), \mu, \mathbf{F})$, where $\mu$ denotes 
the Lebesgue measure, $\mathcal{B}([0,1])$ is the Borel $\sigma$-algebra on $[0,1], \Lambda \subset[0, \infty)$. These spaces are also denoted by $M_{\Lambda, E}$, where $E=L_{p}([0,1], \mathbf{F})$.

Henceforward, measures are considered on Borel $\sigma$-algebras, and for brevity short $\mathcal{B}([a, b])$ will be omitted from the notation of the corresponding Banach spaces.

Henceforth, it is supposed that $\Lambda$ is an increasing sequence contained in $(0, \infty)$ and satisfying the gap condition

$$
\inf _{k}\left\{\lambda_{k+1}-\lambda_{k}\right\}=: \alpha_{0}>0
$$

and the Müntz condition

$$
\sum_{k=1}^{\infty} \frac{1}{\lambda_{k}}=: \alpha_{1}<\infty
$$

Lemma 1. The Müntz space $M_{\Lambda, p}$ is isomorphic with the Banach subspace $M_{\Lambda, p}([0, \infty), v, \mathbf{R}):=c l-$ $\operatorname{span}_{\mathbf{R}}\{\exp (-k x): x \in[0, \infty) ; k \in \Lambda\}$ in the Banach space $L_{p}([0, \infty), \nu, \mathbf{R})$, where $v(d x)=e^{-x} \mu(d x)$.

Proof. The change of the variable $t=\exp (-x)$ gives $\exp (-[0, \infty))=(0,1], d t=-\exp (-x) d x$, $t^{k}=\exp (-k x)$; consequently,

$$
\int_{0}^{1} f(t) g(t) d t=\int_{0}^{\infty} f(\exp (-x)) g(\exp (-x)) \exp (-x) d x
$$

Lemma 2. The Banach space $L_{p}([0, \infty), v, \mathbf{R})$ is isomorphic with $L_{p}([0, \infty), \mu, \mathbf{R})$.

Proof. The measures $\mu$ and $v$ are equivalent; consequently, these Banach spaces are linearly topologically isomorphic (see also $[19,22])$.

Lemma 3. The Banach space $L_{p}([0, \infty), \mu, \mathbf{R})$ is isomorphic with the direct sum of the Banach spaces $\left(\oplus_{m=0}^{\infty} L_{p}([2 \pi m, 2 \pi(m+1)), \mu, \mathbf{R})\right)_{p}$ with the norm inherited from $L_{p}([0, \infty), \mu, \mathbf{R})$.

Proof. For any function $g \in L_{p}([2 \pi m, 2 \pi(m+1)), \mu, \mathbf{R})$, one can take its extension function $g_{m}(x)=$ $g(x)$ for each $x$ in $[2 \pi m, 2 \pi(m+1))$, while $g_{m}(x)=0$ for each $x \in \mathbf{R} \backslash[2 \pi m, 2 \pi(m+1))$. Then, $g_{m}$ is in $L_{p}([0, \infty), \mu, \mathbf{R})$. Therefore, there is an isometric embedding

$$
\theta_{m}: L_{p}([2 \pi m, 2 \pi(m+1)), \mu, \mathbf{R}) \hookrightarrow L_{p}([0, \infty), \mu, \mathbf{R})
$$

for each $m \in \mathbf{N}_{0}$ such that

$$
\theta_{m}(f)=f_{m}
$$

for each $f \in L_{p}([2 \pi m, 2 \pi(m+1)), \mu, \mathbf{R})$. Let $\chi_{A}$ denote the characteristic function of a subset $A$, $\chi_{A}(x)=1$ for each $x \in A$, whereas $\chi_{A}(x)=0$ for any $x \notin A$. As is usual for a sequence of Banach spaces $X_{m}$, the direct sum

$$
X=\left(\bigoplus_{m=0}^{\infty} X_{m}\right)_{p}=l_{p}\left(X_{m}: m \in \mathbf{N}_{0}\right)
$$

denotes the Banach space of all vectors $x=\left(x_{m}: \forall m \in \mathbf{N}_{0} x_{m} \in X_{m}\right)$ of the finite norm

$$
\|x\|_{p}:=\left(\sum_{m=0}^{\infty}\left\|x_{m}\right\|_{X_{m}}^{p}\right)^{1 / p}<\infty .
$$

Thus, the mapping

$$
T(g):=\left(g \chi_{[2 \pi m, 2 \pi(m+1))}: m \in \mathbf{N}_{0}\right)
$$


from $L_{p}([0, \infty), \mu, \mathbf{R})$ is a linear isometry onto $\left(\bigoplus_{m=0}^{\infty} L_{p}([2 \pi m, 2 \pi(m+1)), \mu, \mathbf{R})\right)_{p}$ with the norm inherited from $L_{p}([0, \infty), \mu, \mathbf{R})$ such that

$$
\left(\bigoplus_{m=0}^{\infty} L_{p}([2 \pi m, 2 \pi(m+1)), \mu, \mathbf{R})\right)_{p}=l_{p}\left(L_{p}([2 \pi m, 2 \pi(m+1)), \mu, \mathbf{R}): m \in \mathbf{N}_{0}\right)
$$

where $\mathbf{N}=\{1,2,3, \ldots\}$ and $\mathbf{N}_{0}=\{0\} \cup \mathbf{N}$ (see also [22] or Pelczynsky's decomposition method in [5]).

Next, the Fourier transform is studied in the reflexive Müntz spaces with a change of the variable.

Lemma 4. A continuous linear operator exists, induced by the Fourier sine transform $F_{s}\left(f(x) e^{-x / p}\right)$ from $M_{\Lambda, p}([0, \infty), v, \mathbf{R})$ into $L_{q}(\mathbf{R}, \mu, \mathbf{R})$, where $1 / q+1 / p=1,1<p \leq 2, f \in M_{\Lambda, p}([0, \infty), v, \mathbf{R})$.

Proof. A function $f(x)$ belongs to $M_{\Lambda, p}([0, \infty), v, \mathbf{R})$ if and only if $f(x) e^{-x / p}$ is in $M_{\Lambda, p}([0, \infty), \mu, \mathbf{R})$, since $v(d x)=e^{-x} \mu(d x)$ so that the operator

$$
B_{p}(f)(x):=f(x) e^{-x / p}
$$

is the isometry from $L_{p}([0, \infty), v, \mathbf{R})$ onto $L_{p}([0, \infty), \mu, \mathbf{R})$. We consider the odd extension

$$
f(-x) e^{-|x| / p}=-f(x) e^{-|x| / p}
$$

from $[0, \infty)$ onto $\mathbf{R}$ for each $f \in M_{\Lambda, p}([0, \infty), v, \mathbf{R})$. There is the natural embedding

$$
\psi_{p}: M_{\Lambda, p}([0, \infty), v, \mathbf{R}) \hookrightarrow L_{p}([0, \infty), v, \mathbf{R})
$$

(see Section 2). Then, the sine transform

$$
\begin{gathered}
F_{S}\left(f(x) e^{-x / p}\right)(y):=\sqrt{\frac{2}{\pi}} \int_{0}^{\infty} f(x) e^{-x / p} \sin (y x) d x \\
=\frac{i}{\sqrt{2 \pi}} \int_{-\infty}^{\infty} f(x) e^{-|x| / p} \exp (-i y x) d x
\end{gathered}
$$

is continuous from $L_{p}([0, \infty), \mu, \mathbf{R})$ into $L_{q}(\mathbf{R}, \mu, \mathbf{R})$ according to Theorem 33.5 [23], where $i=\sqrt{-1}$. Indeed, the latter theorem states that if $h \in L_{p}(\mathbf{R}, \mu, \mathbf{C})$, then the sequence of functions

$$
\left\{\frac{1}{\sqrt{2 \pi}} \int_{-n}^{n} h(x) \exp (-i y x) d x: n \in \mathbf{N}\right\}
$$

converges in the $L_{q}(\mathbf{R}, \mathcal{B}(\mathbf{R}), \mu, \mathbf{C})$-norm to a function $F(h) \in L_{q}(\mathbf{R}, \mu, \mathbf{C})$, and the Fourier transform is non-expanding:

$$
\|F(h)\|_{L_{q}(\mathbf{R}, \mu, \mathbf{R})} \leq\|h\|_{L_{p}(\mathbf{R}, \mu, \mathbf{R})} .
$$

At the same time, $F_{s}\left(f(x) e^{-x / p}\right)(y)$ is a real-valued function, since $f$ is real-valued.

Remark 1. Let $M_{\Lambda, p}^{\prime}([0, \infty), v, \mathbf{R})$ denote the topologically dual space of the Müntz space $M_{\Lambda, p}([0, \infty), v, \mathbf{R})$. In the case $1 \leq p \leq 2$, the Fourier transform in $L_{p}$ spaces is defined as usual. For $p>2$, the Fourier transform in $L_{p}$ is understood in the sense of the dual pair $\left(L_{q}, L_{p}\right)$ so that $(F(f), g)=(f, F(g))$ for each $f \in L_{q}$ and $g \in L_{p}$, where $1 / q+1 / p=1$ (see, for example, [23-26] and references therein).

Corollary 1. The Fourier sine transform induces a continuous linear operator from $M_{\Lambda, p}([0, \infty), v, \mathbf{R})$ into $M_{\Lambda, p}^{\prime}([0, \infty), v, \mathbf{R})$. 
Proof. Each continuous linear functional $\phi$ on $M_{\Lambda, p}([0, \infty), v, \mathbf{R})$ has the continuous extension onto $L_{p}([0, \infty), v, \mathbf{R})$ due to the Hahn-Banach extension theorem (see Section 7.1.2 in [3] or (8.4.7) in [15]).

For the dual pair $\left(L_{p}(\mathbf{R}, \mu, \mathbf{C}) ; L_{q}(\mathbf{R}, \mu, \mathbf{C})\right)$ of Banach spaces, a continuous adjoint operator $F^{\prime}$ to $F$ exists (see Proposition 1 and Corollary 6 in Section 8.6 [3]). On the other hand, we infer that

$$
[\phi, \hat{F}(f)]=\left[\hat{F}^{\prime} \phi, f\right],
$$

where $[\phi, f]$ denotes the value of a functional $\phi$ at $f$, where the notation is used

$$
\hat{F}(f):=F_{s}\left(f e^{-x / p}\right),
$$

while $\hat{F}^{\prime}$ stands for the adjoint operator, which exists due to Formulas (8), (11), and (13) in the proof of Lemma 4 above.

\section{The Potential Transform for Reflexive Müntz Spaces}

Lemma 5. For every $f \in L_{p}([0, \infty), v, \mathbf{R})$ and $g \in L_{q}([0, \infty), \mu, \mathbf{R})$, the equality

$$
\int_{0}^{\infty} f(x) e^{-x / p} g(x) \mu(d x)=\int_{0}^{\infty} F_{s}\left(f(x) e^{-x / p}\right)(y) F_{s}(g)(y) \mu(d y)
$$

is satisfied.

Proof. Recall that, on $[0, \infty)$, the measure $v$ has density $e^{-x}$ with respect to the Lebesgue measure $\mu$. The operator $B_{p}$ given by Formula (8) is the linear isometry from $L_{p}([0, \infty), v, \mathbf{R})$ onto $L_{p}([0, \infty), \mu, \mathbf{R})$, since $v(d x) / \mu(d x)=e^{-|x|}$. At the same time, $L_{p}^{\prime}([0, \infty), \mu, \mathbf{R})$ is isomorphic with $L_{q}([0, \infty), \mu, \mathbf{R})$, where $1 / p+1 / q=1$. That is, for each continuous linear functional $g$ on $L_{p}([0, \infty), v, \mathbf{R})$ there exists a function $h \in L_{q}([0, \infty), \mu, \mathbf{R})$ for which $g$ has the form

$$
[g, f]=\int_{0}^{\infty} h(x) f(x) e^{-x / p} \mu(d x)=\left(h, B_{p} f\right),
$$

where

$$
(h, v)=\int_{0}^{\infty} h(x) v(x) \mu(d x)
$$

for any $v \in L_{q}([0, \infty), \mu, \mathbf{R})$, where $\mu$ denotes the Lebesgue measure on the Borel $\sigma$-algebra $\mathcal{B}(\mathbf{R})$ as above. The space $L_{2} \cap L_{p}(\mathbf{R}, \mu, \mathbf{C})$ is dense in $L_{p}(\mathbf{R}, \mu, \mathbf{C})$. The Fourier transform $F$ is the unitary operator from $L_{2}(\mathbf{R}, \mu, \mathbf{C})$ onto $L_{2}(\mathbf{R}, \mu, \mathbf{C})$ such that the Parceval equality is satisfied

$$
\int_{-\infty}^{\infty} h(x) \bar{g}(x) \mu(d x)=\int_{-\infty}^{\infty} F(h)(y) F(\bar{g})(y) \mu(d y)
$$

for every $h, g \in L_{2}(\mathbf{R}, \mu, \mathbf{C})$. Therefore, by continuity, for the dual pair $\left(L_{p}, L_{q}\right)$ this equality is also valid for any $h \in L_{p}(\mathbf{R}, \mu, \mathbf{C})$ and $g \in L_{q}(\mathbf{R}, \mu, \mathbf{C})$, where $\bar{z}$ denotes the conjugated number of a complex number $z \in \mathbf{C}$. Using odd extensions of functions and Formulas (11) and (19), we deduce Equality (16).

Further, the Stieltjes transform and a potential transform are investigated in the reflexive Müntz spaces with a change of the variable.

Proposition 1. There is the identity

$$
\int_{0}^{\infty} \exp (-k x) e^{-x / p} g(x) \mu(d x)=\sqrt{\frac{2}{\pi}} \int_{0}^{\infty} \frac{y F_{s}(g)(y)}{(k+1 / p)^{2}+y^{2}} \mu(d y)
$$


for each $k \in \mathbf{N}_{0}$ and $g \in L_{q}([0, \infty), \mu, \mathbf{R})$.

Proof. This follows from Formula (16), since

$$
F_{S}(\exp (-a x))=\sqrt{\frac{2}{\pi}} \int_{0}^{\infty} \exp (-a x) \sin (y x) d x=\sqrt{\frac{2}{\pi}} \frac{y}{a^{2}+y^{2}}
$$

for each $a>0$ (see Formulas 2-7-2 on page 49 and S7 on page 518 in [27]) and putting $a=k+1 / p$ in the considered case.

Corollary 2. A function $g$ is in the orthogonal complement $\left(M_{\Lambda, p}([0, \infty), v, \mathbf{R})\right)^{\perp}$ of the Müntz space if and only if

$$
\int_{0}^{\infty} \frac{y F_{s}(g)(y)}{(k+1 / p)^{2}+y^{2}} \mu(d y)=0
$$

for each $k \in \Lambda$.

Proof. From Embedding Formulas (10),it follows that for the space $M_{\Lambda, p}([0, \infty), v, \mathbf{R})$ its orthogonal complement $\left(M_{\Lambda, p}([0, \infty), v, \mathbf{R})\right)^{\perp}$ is contained in the topologically dual space $L_{p}^{\prime}([0, \infty), v, \mathbf{R})$ and consists of all $g \in L_{p}^{\prime}([0, \infty), v, \mathbf{R})$ such that

$$
[g, f]=0 \text { for each } f \in M_{\Lambda, p}([0, \infty), v, \mathbf{R})
$$

(see also Section 9.8 in [15] and Formula (17) above). Thus, Formula (21) follows from Identities (16)-(20), the definition of the Müntz space, Lemma 1 and Condition (2), since the real linear span $\operatorname{span}_{\mathbf{R}}\{\exp (-k x): x \in[0, \infty) ; k \in \Lambda\}$ is dense in $M_{\Lambda, p}([0, \infty), v, \mathbf{R})$.

Remark 2. The integral

$$
G(x):=\frac{2}{\pi} \int_{0}^{\infty} \frac{y g(y)}{x^{2}+y^{2}} \mu(d y)
$$

is called the potential transform whenever this integral converges, where $x \in \mathbf{R}$ (see Section 7.2 in [28]). Generally, this integral is considered as the improper integral:

$$
\int_{0}^{\infty} h(y) \mu(d y):=\lim _{\epsilon \downarrow 0} \int_{\epsilon}^{1} h(y) \mu(d y)+\lim _{R \rightarrow \infty} \int_{1}^{R} h(y) \mu(d y)=: \int_{0+}^{\infty} h(y) \mu(d y) .
$$

It is related with the Stieltjes transform by the change of variables $t=y^{2}$ :

$$
2 \int_{0}^{\infty} \frac{y g(y)}{x^{2}+y^{2}} \mu(d y)=\int_{0}^{\infty} \frac{g(\sqrt{t})}{s+t} \mu(d t):=S(h)(s),
$$

where $s=x^{2}, h(t)=g(\sqrt{t})$. In the Stieltjes transform, $S(h)(s)$ generally the complex variable $s=\sigma+i \tau \in \mathbf{C}$ is considered with $\sigma, \tau \in \mathbf{R}$ (see Chapter 7 in [29] or [30]).

Remark 3. Let $\alpha$ be a function of bounded variation on each segment $[0, R]=\{t: 0 \leq t \leq R\}$, where $0<R<\infty$. Then we put

$$
\int_{0}^{\infty} \frac{d \alpha(t)}{(s+t)^{\rho}}:=\lim _{R \rightarrow \infty} \int_{0}^{R} \frac{d \alpha(t)}{(s+t)^{\rho}}
$$

whenever the limit exists, where $\rho>0, s=\sigma+i \tau \in \mathbf{C}, \sigma, \tau \in \mathbf{R}$. 
If a function $\alpha$ is of bounded variation on each segment $[\epsilon, R]=\{t: \epsilon \leq t \leq R\}$, where $0<\epsilon<R<\infty$, and if the limits

$$
A(s)=\lim _{\epsilon \downarrow 0} \int_{\epsilon}^{1} \frac{d \alpha(t)}{(s+t)^{\rho}} \text { and } B(s)=\lim _{R \rightarrow \infty} \int_{1}^{R} \frac{d \alpha(t)}{(s+t)^{\rho}}
$$

both exist for some $s \in \mathbf{C}$, then one writes

$$
f(s)=\int_{0+}^{\infty} \frac{d \alpha(t)}{(s+t)^{\rho}}=A(s)+B(s) .
$$

Next, we shall use Theorems 13, 14.2 from [28], 2a, 2b, and $7 \mathrm{~b}$ from $[29,30]$ about the potential and Stieltjes transforms.

Lemma 6. Suppose that $g$ is an odd function on $\mathbf{R}$ such that $g \in L_{q}(\mathbf{R}, \mu, \mathbf{R})$ and its support is contained in $[-2 \pi(m+1),-2 \pi m] \cup[2 \pi m, 2 \pi(m+1)]$ for some nonnegative integer $m$, and $F_{s}(g)(y)$ is continuous in the variable $y \in \mathbf{R}$, where $1<q<\infty$. Then, its Fourier sine transform coefficients $b_{n}(g)$ are given by the formula:

$$
b_{n}(g)=\lim _{\eta \downarrow 0} \frac{S(h)(-n-i \eta)-S(h)(-n+i \eta)}{2 \pi i}
$$

for each $n \in \mathbf{N}$, where $h(t)=F_{s}(g)(\sqrt{t})$ while $t \geq 0$.

Proof. Formula (28) follows from Theorem $7 \mathrm{~b}$ [29] and Identity (25).

Proposition 2. Let $g \in M_{\Lambda, p}^{\prime}([0, \infty), \mu, \mathbf{R})$ and $g_{m}=g \chi_{[2 \pi m, 2 \pi(m+1))}$ and let $G_{m}(s)$ be a continuous function such that

$$
G_{m}(s)=\int_{0}^{\infty} \exp (-x(k+1 / p)) g_{m}(x) \mu(d x)
$$

for each $m \in \mathbf{N}_{0}$, where $1<p<\infty, s=(k+1 / p)^{2}, k \in[0, \infty)$. Then, up to the isomorphism of the Müntz spaces, the sine Fourier coefficients $b_{n}\left(g_{m}\right)$ of $g_{m}$ are given by the formula:

$$
b_{n}\left(g_{m}\right)=F_{\mathcal{S}}\left(g_{m}\right)(n)=\lim _{\eta \downarrow 0} \frac{G_{m}(-n-i \eta)-G_{m}(-n+i \eta)}{2 \pi i}
$$

for every $n \in \mathbf{N}$. If in addition

$$
G(s)=\int_{0}^{\infty} \exp (-x(k+1 / p)) g(x) \mu(d x)
$$

is continuous, then

$$
F_{S}(g)(n)=\sum_{m=0}^{\infty} F_{s}\left(g_{m}\right)(n)=\lim _{\eta \downarrow 0} \frac{G(-n-i \eta)-G(-n+i \eta)}{2 \pi i} .
$$

Proof. For each function $g \in L_{q}(\mathbf{R}, \mu, \mathbf{R})$, its restriction $u_{n}=g \chi_{[2 \pi n, 2 \pi(n+1))}$ has a Fourier series decomposition, so that

$$
\lim _{m \rightarrow \infty}\left\|S_{m}\left(u_{n}\right)-u_{n}\right\|_{L_{q}}=0
$$

due to the Riesz theorem (see [31]), where $1 / p+1 / q=1, n \in \mathbf{Z}$,

$$
S_{m}(u)(x)=\sum_{k=-m}^{m} a_{k} e^{i k x}
$$


denotes the partial sum of the Fourier series, $a_{k}=a_{k}(u)$ are decomposition coefficients of $u$. Moreover, in view of the Hunt theorem if $u \in L_{p}([0,2 \pi])$ for some $1<p<\infty$, then

$$
\left\|S^{*}\right\|_{L_{p}} \leq A(p)\|u\|_{L_{p}}
$$

where $A(p)>0$ is a constant independent of $u$, where

$$
S^{*}(x, u):=\sup _{m \in \mathbf{N}}\left|S_{m}(u)(x)\right|
$$

(see [31]). Certainly, Fourier coefficient functionals $a_{k}$ are continuous from $L_{p}([0,2 \pi), \mu, \mathbf{R})$ into $\mathbf{C}$ due to the integral Hölder inequality:

$$
\int_{b}^{c}|f(t) g(t)| d t \leq\left(\int_{b}^{c}|f(t)|^{p}\right)^{1 / p}\left(\int_{b}^{c}|g(t)|^{q}\right)^{1 / q},
$$

where

$$
a_{k}(u):=\frac{1}{\sqrt{2 \pi}} \int_{0}^{2 \pi} u(t) e^{-i k t} d t
$$

$i=\sqrt{-1}, k \in \mathbf{Z}, f \in L_{p}([b, c]), g \in L_{q}([b, c])$ with the Lebesgue measure considered on the segment $[b, c]$.

According to Lemma 2.2 in [8], the Müntz space $M_{\Lambda, p}([0,1], \mu, \mathbf{R})$ is isomorphic to the one with $\Lambda_{1}:=\delta+\gamma \Lambda \subset \mathbf{N}-1 / p$ and by Lemma 1 with $M_{\Lambda_{1}, p}([0, \infty), v, \mathbf{R})$. Therefore, their topologically dual spaces $M_{\Lambda, p}^{\prime}([0,1], \mu, \mathbf{R})$ and $M_{\Lambda_{1}, p}^{\prime}([0, \infty), v, \mathbf{R})$ are also isomorphic; hence, Formula (30) follows from Proposition 1 and Lemma 6. Moreover, if $G(s)$ is continuous, then by Theorem $7 \mathrm{~b}$ [29] and Proposition 1, the following limit exists:

$$
F_{s}(g)(n)=\lim _{\eta \downarrow 0} \frac{G(-n-i \eta)-G(-n+i \eta)}{2 \pi i} .
$$

By virtue of the Hahn-Banach extension Theorem (8.4.7) in [15], each continuous linear functional $g$ on $M_{\Lambda, p}([0, \infty), v, \mathbf{R})$ has a continuous extension onto $L_{p}([0, \infty), v, \mathbf{R})$. On the other hand, the series $\sum_{m} g_{m}$ converges to $g$ in the Banach space $M_{\Lambda_{1}, p}^{\prime}([0, \infty), v, \mathbf{R})$ relative to the $L_{q}$ norm, since $L_{p}^{\prime}=L_{q}$ with $1 / p+1 / q=1$. First observe that the function $\exp (-x / p)$ belongs to the space $L_{p}([0, \infty), \mu, \mathbf{R})$. Then, from the Hölder inequality

$$
\begin{gathered}
\left|\int_{0}^{\infty} \exp (-x(k+1 / p))\left[\sum_{m=0}^{n} g_{m}(x)-g(x)\right] \mu(d x)\right| \leq \\
\|\exp (-x(k+1 / p))\|\left\|_{L_{p}}\right\| \sum_{m=0}^{n} g_{m}(x)-g(x)\left\|_{L_{q}} \leq\right\| \exp (-x / p)\|\|_{L_{p}}\left\|\sum_{m=0}^{n} g_{m}(x)-g(x)\right\|_{L_{q}}
\end{gathered}
$$

for each $k \geq 0$ it follows that

$$
\lim _{n \rightarrow \infty} \sum_{m=0}^{n} G_{m}(s)=G(s) \text { for each } s \in\left[p^{-2}, \infty\right),
$$

since $0<\exp (-x(k+1 / p)) \leq \exp (-x / p)$ for each $k \geq 0$ and $x \in[0, \infty)$.

Using the preceding results, a geometry of the reflexive Müntz spaces is investigated below. 


\section{Isomorphic Classification}

Proposition 3. Let $f$ be a continuous linear functional on $L_{p}([0, \infty), \mu, \mathbf{R})$ with $1<p<\infty$, let also $f$ be presented by a function $f \in \mathcal{S}([0, \infty))$. Then, it satisfies the condition:

$$
\left[F_{\mathcal{S}}(f)\right](\lambda)=0
$$

for each $\lambda \in \Lambda+1 / p$ if and only if its kernel $\operatorname{ker}(f)$ contains the Müntz space $M_{\Lambda, p}([0, \infty), \mu, \mathbf{R})$.

Proof. Recall that $\mathcal{S}([0, \infty))$ denotes a space of all infinite differentiable functions $h:[0, \infty) \rightarrow \mathbf{R}$ such that $\lim _{x \rightarrow \infty} x^{n} D^{m} h(x)=0$ for all nonnegative integers $n$ and $m$. As is traditional, the space $\mathcal{S}([0, \infty))$ is supplied with the family of semi-norms $\rho_{n, m}(h):=\sup _{x \in[0, \infty)}\left|x^{n} D^{m} h(x)\right|<\infty$, where $n$ and $m$ are nonnegative integers.

Since $f$ provides a continuous linear functional on $L_{p}([0, \infty), \mu, \mathbf{R})$ with $1<p<\infty$, and since the topological dual space of $L_{p}$ is $L_{q}$, where $1 / p+1 / q=1$, then the function $f$ belongs to $L_{q}([0, \infty), \mu, \mathbf{R})$. This means that the value $\left\langle g, f>\right.$ of $f$ at $g \in L_{p}$ is given by

$$
<g, f>=\int_{0}^{\infty} g(x) f(x) d x
$$

Without loss of generality for each function $g \in L_{r}([0, \infty), \mu, \mathbf{R})$ with $1 \leq r<\infty$ we can consider the identically zero extension of $g$ onto $(-\infty, 0)$. The Fourier transform is defined on $L_{r}(\mathbf{R}, \mu, \mathbf{R})$ for $1 \leq r \leq 2$ as:

$$
F(h)(x):=\frac{1}{\sqrt{2 \pi}} \lim _{r \rightarrow \infty} \int_{-r}^{r} h(t) e^{-i x t} d t
$$

where $i=\sqrt{-1}, x \in \mathbf{R}$.

The linear span of functions $\exp (-x(k+1 / p))$ with $k \in \Lambda$ is dense in $M_{\Lambda, p}([0, \infty), \mu, \mathbf{R})$. Therefore, $\left\langle f, g>=0\right.$ for each $g \in M_{\Lambda, p}([0, \infty), \mu, \mathbf{R})$ if and only if $<f, \exp (-x(k+1 / p))>=0$ for all $k \in \Lambda$.

The Fourier transform is a unitary operator on $L_{2}$, and by the duality has the weakly continuous extension for the dual pair $<L_{p}, L_{q}>$ such that the Parseval identity is satisfied

$$
<F g, F f>=<g, f>
$$

for each $g \in L_{p}$ and $f \in L_{q}$ (see [24,25]). The sine Fourier transform $F_{\mathcal{S}}$ maps $\mathcal{S}([0, \infty))$ into itself so that

$$
\sum_{m=0}^{\infty}\left[F_{\mathcal{S}}\left(f \chi_{[2 \pi m, 2 \pi(m+1))}\right)\right]=F_{\mathcal{S}}(f)
$$

for any $f \in \mathcal{S}([0, \infty))$. Since $f \in \mathcal{S}([0, \infty))$, the function $g=f$ satisfies conditions of Proposition 2 . Thus, Formula (38) follows from Formulas (30)-(32).

Proposition 4. The second orthogonal $\left(M_{\Lambda_{, p}}^{\perp}\right)^{\perp}([0, \infty), \mu, \mathbf{R})$ of the Müntz space $M_{\Lambda, p}([0, \infty), \mu, \mathbf{R})$ in $L_{p}([0, \infty), \mu, \mathbf{R})$ with $1<p<\infty$ is characterized by the condition:

$$
\int_{0}^{\infty} f(x) g(x) d x=0
$$

for each $f$ satisfying the conditions of Proposition 3 and each $g \in\left(M_{\Lambda, p}^{\perp}\right)^{\perp}([0, \infty), \mu, \mathbf{R})$.

Proof. Since the Müntz space $X=M_{\Lambda, p}([0, \infty), \mu, \mathbf{R})$ is the linear subspace in the normed space $Y$, the Hahn-Banach Theorem (8.4.7) in [15] implies that each continuous linear functional $f$ on $X$ has a continuous linear extension to $Y=L_{p}([0, \infty), \mu, \mathbf{R})$. It follows that $M_{\Lambda, p}^{\perp}([0, \infty), \mu, \mathbf{R})$ is a Banach 
space in which the linear subspace of all functionals satisfying the conditions of Proposition 3 is dense.

For all functions $h \in L_{q}([0, \infty), \mu, \mathbf{R})$ and $u \in L_{p}([0, \infty), \mu, \mathbf{R})$ with $1 / p+1 / q=1$, the Hölder inequality

$$
\left|\int_{0}^{\infty} u(x) h(x) d x\right| \leq\|u\|_{L_{p}}\|h\|_{L_{q}}
$$

is fulfilled. Since the subspace $\mathcal{S}([0, \infty))$ is dense in the Banach space $L_{q}([0, \infty))$ relative to the $L_{q}$ norm topology and $F_{\mathcal{S}}(\mathcal{S}([0, \infty))=\mathcal{S}([0, \infty))$, then from Inequality (43) it follows that if (42) is satisfied for a function $g \in L_{p}$ for each $f \in \mathcal{S}([0, \infty))$ satisfying Condition (38), then $g \in\left(M_{\Lambda, p}^{\perp}\right)^{\perp}([0, \infty), \mu, \mathbf{R})$. Thus, $g \in\left(M_{\Lambda, p}^{\perp}\right)^{\perp}([0, \infty), \mu, \mathbf{R})$ if and only if Formula (42) is valid for all $f$ satisfying the conditions of Proposition 3.

Theorem 1. Suppose that an increasing sequence $\Lambda$ satisfies the gap and Müntz conditions (2) and (3). Let $1<p<\infty$. Then the Müntz space $M_{\Lambda, p}([0,1], \mu, \mathbf{F})$ is isomorphic with $l_{p}(\mathbf{F})$.

\section{Proof.}

(I). In view of Theorem 2.1 and Lemma 2.2 in [8], up to the Banach spaces isomorphism it is sufficient to consider the case $\Lambda \subset \mathbf{N}$. It is clear that if the theorem is proved over the real field $\mathbf{R}$, then from it the case over the complex field $\mathbf{C}$ follows.

(II). The Banach space $L_{p}(\mathbf{R}, \mu, \mathbf{R})$ is isomorphic with the Banach space

$$
l_{p}\left(L_{p}([2 \pi n, 2 \pi(n+1)), \mu, \mathbf{R}): n \in \mathbf{Z}\right) .
$$

Let $L_{p, o}(\mathbf{R}, \mu, \mathbf{R})$ denote the Banach subspace consisting of all odd functions in $L_{p}(\mathbf{R}, \mu, \mathbf{R})$. Then, we put $L_{p, o}([2 \pi n, 2 \pi(n+1)), \mu, \mathbf{R})$ to be the Banach subspace of all functions $g \in L_{p}([2 \pi n, 2 \pi(n+1))$, $\mu, \mathbf{R})$ such that $g(2 \pi n+x)=-g(2 \pi(n+1)-x)$ for almost each $x \in[0, \pi]$. Therefore, the Banach space $L_{p, o}(\mathbf{R}, \mu, \mathbf{R})$ is isomorphic with $l_{p}\left(L_{p, o}([2 \pi n, 2 \pi(n+1)), \mu, \mathbf{R}): n \in \mathbf{Z}\right)$ and with $L_{p}([0, \infty), \mu, \mathbf{R})$.

In view of Theorem 33.3 [23], if $1<p \leq 2$ and $1 / p+1 / q=1, f \in L_{p}([2 \pi n, 2 \pi(n+1)), \mu, \mathbf{R})$ and if $\hat{a}(f):=\left\{a_{n}(f): n \in \mathbf{Z}\right\}$ is the sequence of Fourier coefficients of $f$, then $\hat{a}(f) \in l_{q}$ and

$$
\|\hat{a}(f)\|_{l_{q}} \leq\|f\|_{L_{p}}
$$

where

$$
l_{q}=l_{q}(\mathbf{C}, \mathbf{Z}):=\left\{x=\left(x_{k}: x_{k} \in \mathbf{C}, k \in \mathbf{Z}\right) ;\|x\|_{l_{q}}:=\left[\sum_{k=-\infty}^{\infty}\left|x_{k}\right|^{q}\right]^{1 / q}<\infty\right\} .
$$

Moreover, Theorem 33.4 [23] asserts that if $1<p \leq 2$ and $1 / p+1 / q=1$, if $\hat{b}=\left\{b_{n}: b_{n} \in \mathbf{C}\right.$, $n \in \mathbf{Z}\} \in l_{p}$ is given and

$$
S_{m}(x):=\sum_{k=-m}^{m} b_{k} e^{i k x}
$$

is the $m$-th partial sum of the trigonometric series $\sum_{k=-\infty}^{\infty} b_{k} e^{i k x}$, then the sequence of sums $S_{m}(x)$ tends to a function $f \in L_{q}([0,2 \pi), \mu, \mathbf{C})$ in the $L_{q}$ norm as $m$ tends to infinity; furthermore,

$$
\hat{b}=\hat{a}(f) \text { and }\|f\|_{L_{q}} \leq\|\hat{b}\|_{l_{p}} .
$$

For odd real-valued functions, we consider the partial sums of the form

$$
S_{m, o}(x)=\sum_{k=1}^{m} a_{k} \sin (k x),
$$


where $a_{k} \in \mathbf{R}$. On the other hand, there exists an isomorphism of $l_{q} \oplus l_{q}$ with $l_{q}$. Therefore, there exists a linear projection operator $E$ from $l_{q}(\mathbf{R})$ onto the space

$$
Q:=\left\{x \in l_{q}(\mathbf{R}): \forall k \in \mathbf{N} \backslash \Lambda \quad x_{k}=0\right\},
$$

where $\Lambda \subset \mathbf{N}$. At the same time, there exists the continuous projection linear operator $A$ from $l_{q}\left(l_{q}(\mathbf{R}), \mathbf{N}\right)$ onto

$$
V:=\left\{x \in l_{q}\left(l_{q}(\mathbf{R}), \mathbf{N}\right): \forall k \in \mathbf{N} \backslash \Lambda \forall n \in \mathbf{N}_{0} \quad x_{k}(n)=0\right\},
$$

where

$$
\begin{gathered}
l_{q}\left(J_{n}: n \in \mathbf{N}\right):= \\
\left\{x=\left(x(n): x(n) \in J_{n}, n \in \mathbf{N}\right) ;\|x\|_{l_{q}}:=\left[\sum_{n=1}^{\infty}\|x(n)\|_{J_{n}}^{q}\right]^{1 / q}<\infty\right\}
\end{gathered}
$$

for a sequence of Banach spaces $J_{n}$ over the same field either $\mathbf{R}$ or $\mathbf{C}$, while $l_{q}\left(J, \mathbf{N}_{0}\right):=l_{q}\left(J_{n}\right.$ : $\left.J_{n}=J, n \in \mathbf{N}_{0}\right)$, where $\mathbf{N}=\{1,2,3, \ldots\}, \mathbf{N}_{0}=\mathbf{N} \cup\{0\}$.

Its adjoint operator $A^{\prime}$ relative to the dual pair $\left(l_{q}, l_{p}\right)$ is the continuous embedding from $l_{p}\left(l_{p}, \mathbf{N}_{0}\right)$ into $l_{p}\left(Q^{\prime}, \mathbf{N}_{0}\right)$ due to Corollary 8.6.4 in [26] and Formulas (47)-(49), since $A^{2}=A$ and hence $\left(A^{\prime}\right)^{2}=\left(A^{2}\right)^{\prime}=A^{\prime}$, where $1<p<\infty$ and $1 / p+1 / q=1$.

By virtue of Theorem (9.11.1) in [15], if $M$ is a closed subspace of a topological vector space $X$, then $(X / M)^{\prime}$ is algebraically isomorphic to $M^{\perp}$, where $X^{\prime}$ denotes the topological dual space of $X$. In view of Theorem (9.11.3) in [15], if $X$ and $Y$ are paired topological vector spaces and $M$ is a closed subspace in $X$, then $X / M$ and $M^{\perp}$ are paired spaces and $\sigma\left(X / M, M^{\perp}\right)$ is the quotient topology on $X / M$ induced by $\sigma(X, Y)$.

Let $\mathbf{S}$ denote the operator from $l_{q}(\mathbf{R})$ into $L_{p, o}([0,2 \pi], \mu, \mathbf{R})$ such that

$$
\mathbf{S} \hat{a}=\sum_{k=1}^{\infty} a_{k} \sin (k x)
$$

where $1<q \leq 2,1 / p+1 / q=1$. We consider its extension from $V$ into $L_{p}([0, \infty), \mu, \mathbf{R})$ :

$$
\mathbf{S}\left(\hat{a}(n): n \in \mathbf{N}_{0}\right)=\sum_{n=0}^{\infty} \sum_{k=1}^{\infty} a_{k}(n) \sin (k x) \chi_{[2 \pi n, 2 \pi(n+1))}(x) .
$$

If $B: H_{0} \rightarrow K$ is a continuous linear operator from the normed space $H_{0}$ into the Banach space $K$, while $H_{0}$ is a dense linear subspace in a normed space $H$ such that the norm on $H_{0}$ is inherited from $H$, then $B$ has a continuous extension from $H$ into $K$ [15].

In view of Lemmas $1-3$ and the isomorphism of the Banach space $L_{p, o}(\mathbf{R}, \mu, \mathbf{R})$ with $l_{p}\left(L_{p, o}([2 \pi n, 2 \pi(n+1)), \mu, \mathbf{R}): n \in \mathbf{Z}\right)$ (see the proof above), there exists the Banach space embedding

$$
\theta: M_{\Lambda, p}([0, \infty), \mu, \mathbf{R}) \hookrightarrow Y:=l_{p}\left(L_{p, o}([2 \pi n, 2 \pi(n+1)), \mu, \mathbf{R}): n \in \mathbf{Z}\right) .
$$

Using Formulas (44), (47), (49), (51), and (52), the dual pairs $\left(L_{p}, L_{q}\right)$ and $\left(l_{p}, l_{q}\right)$, and also the theorems cited above, we deduce that $\left(\theta\left(M_{\Lambda, p}([0, \infty), \mu, \mathbf{R})\right)^{\perp}=: X\right.$ is complemented in $Y^{\prime}$. Therefore, the second orthogonal $\left(M_{\Lambda, p}([0, \infty), \mu, \mathbf{R})\right)^{\perp \perp}$ of the Müntz space $M_{\Lambda, p}([0, \infty), \mu, \mathbf{R})$ is isomorphic with the orthogonal complement subspace $X^{\perp}$ of the topologically complemented subspace $X$ in $Y^{\prime}$.

(III). In view of Theorems 9.2.2 and 9.8.1 in [15], we have that

$$
\left(M_{\Lambda, p}([0, \infty), \mu, \mathbf{R})\right)^{\perp \perp}=M_{\Lambda, p}([0, \infty), \mu, \mathbf{R}),
$$


since the Müntz space $M_{\Lambda, p}([0, \infty), \mu, \mathbf{R})$ is complete and linear. By virtue of the part (II) above, Lemmas 1 and 2 the Müntz space $M_{\Lambda, p}([0,1], \mu, \mathbf{R})$ is complemented in $L_{p}([0,1], \mu, \mathbf{R})$, since the Banach space $L_{p}([0,1], \mu, \mathbf{R})$ is reflexive for $1<p<\infty$. There are natural embeddings of $l_{p}$ into $L_{p}$.

Theorem 9.1.6 [1] asserts that the Müntz space $M_{\Lambda, p}([0,1], \mu, \mathbf{R})$ is isomorphic to a subspace of $l_{p}$, since conditions (2) and (3) are fulfilled and $1<p<\infty$. However, $l_{p}$ is a prime space, which, by definition, means that its infinite dimensional topologically linearly complemented subspace is isomorphic to itself (see volume 1, page 57 and further in [5]). Therefore, the Müntz space $M_{\Lambda, p}([0,1], \mu, \mathbf{R})$ being infinite dimensional, is isomorphic with $l_{p}(\mathbf{R})$.

\section{Conclusions}

The results of this paper can be used not only in Banach space geometry, function approximations, but also for periodic function analysis of perturbations to almost periodic functions with trend [32], also for distortions in high-frequency pulse acoustic signals [33].

Acknowledgments: The author is sincerely grateful to Wolfgang Lusky for discussions.

Conflicts of Interest: The author declares no conflict of interest.

\section{References}

1. Gurariy, V.I.; Lusky, W. Geometry of Müntz spaces and related questions. In Lecture Notes in Mathematics; Springer: Berlin, Germany, 2005.

2. Gurariy, V.I. Bases in spaces of continuous functions on compacts and some geometrical questions. Math. USSR Izvestija 1966, 30, 289-306.

3. Jarchow, H. Locally Convex Spaces; Teubner, B.G., Ed.; Springer: Stuttgart, Germany, 1981.

4. Ludkowski, S.V.; Lusky, W. On the geometry of Müntz spaces. J. Funct. Spaces 2015, 1-7, doi:10.1155/2015/787291.

5. Lindenstrauss, J.; Tzafriri, L. Classical Banach Spaces I and II; A Series of Modern Surveys in Mathematics; Springer-Verlag: Berlin, Germany, 1979.

6. Ludkovsky, S.V. -normed topological vector spaces. Sibirskii Matematicheskii Zhurnal 2000, 41, 141-154.

7. Ludkovsky, S.V. Duality of $\kappa$-normed topological vector spaces and their applications. J. Math. Sci. 2009, 157, 367-385.

8. Ludkowski, S.V. Approximation in Müntz spaces $M_{\Lambda, p}$ of $L_{p}$ functions for $1<p<\infty$ and bases. Mathematics 2017, 5, 10, doi:10.3390/math5010010.

9. Ludkowski, S.V. Approximation and existence of Schauder bases in Müntz spaces of $L_{1}$ functions. J. Math. Anal. Appl. 2016, 441, 635-647, doi:10.1016/j.jmaa.2016.03.064.

10. Lusky, W. On Banach spaces with the commuting bounded approximation property. Arch. Math. 1992, 58, 568-574.

11. Lusky, W. On Banach spaces with bases. J. Funct. Anal. 1996, 138, 410-425.

12. Lusky, W. Three space properties and basis extensions. Isr. J. Math. 1998, 107, 17-27.

13. Lusky, W. Three space problems and bounded approximation property. Stud. Math. 2003, 159, 417-434.

14. Lusky, W. On Banach spaces with unconditional bases. Isr. J. Math. 2004, 143, 239-251.

15. Narici, L.; Beckenstein, E. Topological Vector Spaces; Marcel Dekker, Inc.: New York, NY, USA, 1985.

16. Schauder, J. Zur Theorie stetiger Abbildungen in Funktionalraumen. Math. Z. 1927, 26, 47-65.

17. Schwartz, L. Étude des Sommes D'exponentielles, 2-éme ed.; Hermann: Paris, France, 1959.

18. Semadeni, Z. Schauder Bases in Banach Spaces of Continuous Functions; Springer: Berlin, Germany, 1982.

19. Wojtaszczyk, P. Banach Spaces for Analysts; Cambridge Studies in Advanced Mathematics; Cambridge University Press: Cambridge, UK, 1991.

20. Almira, J.M. Müntz type theorems I. Surv. Approx. Theory 2007, 3, 152-194.

21. Clarkson, J.A.; Erdös, P. Approximation by polynomials. Duke Math. J. 1943, 10, 5-11.

22. Christensen, J.P.R. Topology and Borel Structure; North-Holland Mathematics Studies 10; Elsevier: Amsterdam, The Netherlands, 1974.

23. Zaanen, A.C. Continuity, Integration and Fourier Theory; Springer: Berlin, Germany, 1989. 
24. Bremermann, H. Distributions, Complex Variables, and Fourier Transforms; Addison-Wesley Publ. Co., Inc.: Boston, MA, USA, 1965.

25. Butzer, P.L.; Nessel, R.J. Fourier Analysis and Approximation; Birkhäuser: Basel, Switzerland, 1971.

26. Edwards, R.E. Functional Analysis; Holt, Rinehart and Winston: New York, NY, USA, 1965.

27. Sneddon, I.H. The Use of Integral Transforms; McGraw Hill Book Co.: New York, NY, USA, 1972.

28. Widder, D.V. An Introduction to Transform Theory; Academic Press: New York, NY, USA, 1971.

29. Widder, D.V. The Laplace Transform; Princeton University Press: Princeton, NJ, USA, 1972.

30. Widder, D.V. The Stieltjes transform. Trans. Am. Math. Soc. 1938, 43, 7-60.

31. Zhizhiashvili, L. Trigonometric Fourier Series and Their Conjugates; Mathematics and Its Applications; Kluwer: Dordrecht, The Netherlands, 1996.

32. Kuzmin, V.I.; Samokhin, A.B. Almost periodic functions with trend. Russ. Technol. J. 2015, 2, 105-107.

33. Denisov, V.E. Analysis of distortions in high-frequency pulse acoustic signals with linear frequency modulation in a hydroacoustic communication channels. Russ. Technol. J. 2017, 4, 34-41.

(C) 2017 by the author. Licensee MDPI, Basel, Switzerland. This article is an open access article distributed under the terms and conditions of the Creative Commons Attribution (CC BY) license (http:/ / creativecommons.org/licenses/by/4.0/). 